A patient questionnaire gained the opinions of 38 patients on being weighed and their understanding of the reason for being weighed.

Results $97 \%$ of patients did not find being weighed distressing. However, $51 \%$ of staff members were opposed to routine weighing.

$13 \%$ of patients had a weight recorded. $13 \%$ were prescribed low molecular weight heparin, $80 \%$ of these patients were weighed and $60 \%$ were on the correct dose.

Implications Routine weighing has been introduced for all patients where appropriate. Clinical staff now receive training that demonstrates the inaccuracy of estimating body weight. An alert sticker is now attached to the medicine chart, for patients prescribed weight dependant medication and a prompt on the shelves where the medication is stored acts as a reminder to check body weight.

\section{P-78 USE OF AUDIT IN MEDICINE MANAGEMENT AT ST ANN'S HOSPICE}

Jan Codling, Kath Mitchell, Jennie Pickard, David Waterman, Elaine Sigsworth, Suzie Doe. St Ann's Hospice, Cheshire, UK

\subsection{6/bmjspcare-2017-00133.77}

Background Medication errors can lead to patient harm including death. Prescribing error rates of $7 \%$ and administration errors of $8 \%$ are recognised. Effective systems and processes can minimise the risk of preventable medicine-related problems.

Methods A four monthly audit of prescribing standards contained in the hospice medicine policy was undertaken by the hospice pharmacists. Prescribers received feedback verbally and via posters.

An annual administration of medicines audit was conducted by the practice development nurses. Nurses received feedback and an action plan was agreed.

During the period April 2015 to June 2016, the hospice introduced the Medicine Safety Thermometer (MST) to assess recording of allergy status, pharmacy medicines reconciliation, omitted medicines and safety of high risk medicines.

Results Audit results are displayed in the clinical areas to highlight the current issues. Findings were also used to inform changes in the medicine chart.

An anonymous questionnaire to doctors showed the prescribing audit was felt to be a useful educational tool.

An action from the MST included the development of a variance recording form, integrated in the medicine chart. This records details why a medication was omitted rather than just using a variance code. For example a patient may decline a medicine because they don't like the taste. The extra detail should trigger an action to resolve the issue.

Implications Prescribing and administration audits and the MST were used in the in-patient hospice environment to identify medicine-related safety incidents. Subsequent learning contributed to the safer use of medicines.

\section{P-79 DISTRESS VERSUS HARM; HAVE WE IMPLEMENTED CHANGES TO DNACPR DOCUMENTATION FOLLOWING THE TRACEY JUDGMENT?}

${ }^{1,2}$ Stephanie Shayler, 'Mike Macfarlane, 'Derek Willis. 'Severn Hospice, Telford, Warwick, UK: ${ }^{2}$ St Marys Hospice, Birmingham, UK

\subsection{6/bmjspcare-2017-00133.78}

Background Following the Tracey judgment in 2014, DNACPR decisions must now be communicated to the patient or, when this is not possible, their relatives. The only exceptions are if the patient has expressed a clear wish not to be involved or there is significant risk of causing physical or psychological harm to the patient by communicating the information.

Currently there is no guidance on what constitutes 'physical or psychological harm', therefore it is subject to varying interpretation.

The aim of this pilot was to investigate the communication of DNACPR decisions following the Tracey case and the interpretation of 'physical or psychological harm' by healthcare professionals.

Methods A retrospective audit of clinical notes was performed. 30 notes were analysed from 2013 (before the Tracey ruling) to determine who DNACPR decisions were communicated to and, if this information was withheld, the reasons why. 30 patient notes from 2015 (following the Tracey ruling) were analysed to obtain the same information, then a comparison was made between both years.

Results 6/30 (20\%) DNACPR decisions were discussed with patients in 2013 compared to $17 / 30$ (57\%) in 2015. 4/30 (13\%) decisions were discussed with families in 2013 compared to $17 / 30(57 \%)$ in 2015 .

Reasons for not discussing DNACPR decisions in 2013: distress (79\%); patient choice (13\%); no reason documented (4\%); anxiety (4\%).

Reasons for not discussing DNACPR discussions in 2015: psychological harm (39\%); no reason documented (23\%); patient choice (15\%); patient confused (15\%) patient unable to communicate $(8 \%)$

Psychological harm in 2015 was described as 'extreme distress', 'anxiety', 'distress', 'extreme distress' and 'upset'.

Conclusions Communication of DNACPR decisions increased following the Tracey judgment.

There was no clear consensus on what constitutes 'harm' although the term 'distress' was most commonly included in its explanation. This indicates the need for further research and guidance in this area.

\section{P-80 PATIENTS WITH METASTATIC CANCER: HOSPICE PATIENTS DIE; HOSPITAL PATIENTS SURVIVE - TRUE OR FALSE?}

\footnotetext{
${ }^{1,2}$ Sanjay Shah. ${ }^{1}$ Northamptonshire Healthcare NHS Foundation Trust, Kettering, UK. ${ }^{2}$ Kettering General Hospital NHS Foundation Trust
}

10.1136/bmjspcare-2017-00133.79

Background No evidence could be found to support the general perception that hospice patients die whereas hospital palliative care patients survive. Such a perception could make patients reluctant to accept hospice support; and lead clinicians to over treat hospital patients and deny beneficial interventions to hospice patients. 
Aims and objectives Compare 3 month survival of patients with metastatic cancer admitted to hospice and hospital.

Methods All adults known to have metastatic cancer preadmission to district general hospital or hospice over a 2 month period were included. Parameters compared: proportions discharged and died; length of in-patient stay; mean survival; survival at 3 months.

Results Total number of patients - 106 (hospital:72; hospice:34). $60 \%$ of hospital group women; $65 \%$ of hospice men. Hospice patients were significantly younger, mean age 69.09 vs 75.36 years; p-value 0.006. There was no difference in baseline investigation (FBC; renal and liver functions; serum calcium) results of two groups. There was no difference in proportion of patients discharged $(57 \%$ hospital vs $59 \%$ hospice); proportion dying during admission (43\% hospital vs $41 \%$ hospice); mean or median length of in-patient stay (hospital vs hospice, mean days: 13.29 vs 14.26 , median -9.5 vs 9.5 ); and mean survival (22 days) in 2 groups. Forty four percent of hospital patients were alive at 3 months, versus $35 \%$ of hospice - but insignificant difference (p-value: 0.121). There was no correlation between age and survival $(r=-0.10$; p-value: 0.941).

Conclusions This small study demonstrates no survival benefit of invasive (district general) hospital approach against holistic hospice approach in patients with metastatic cancer. Hospital clinicians should not be concerned that hospice approach would shorten survival of their patients and lengthen hospital stay. All clinicians and managers should be aware that a third of hospice patients survive more than 3 months and that there are mechanisms to identify and manage them promptly.

\section{P-81 GLASGOW PROGNOSTIC SCALE: USEFULNESS IN PREDICTING 3-MONTH SURVIVAL IN METASTATIC CANCER PATIENTS}

\footnotetext{
${ }^{1,2}$ Sanjay Shah. ${ }^{1}$ Northamptonshire Healthcare NHS Foundation Trust, Kettering, UK; ${ }^{2}$ Kettering General Hospital NHS Foundation Trust
}

\subsection{6/bmjspcare-2017-00133.80}

Background In England, many end of life care or hospice-athome services will provide care for patients with life expectancy of $<8-12$ weeks. Low serum albumin has been associated with poor survival in carcinoma of stomach. Raised serum C-reactive protein (CRP) levels have also been linked to poor survival in cancer patients. Glasgow Prognostic Score (GPS) combines serum albumin and CRP levels to give a composite score. Poor survival is associated with high GPS in colorectal cancer patients.

Aims and objectives To compare 3 month survival of patients with metastatic cancer using GPS

Methods All adults known to have metastatic cancer preadmission to district general hospital and hospice over a 2 month period were included. Using serum albumin $(>35 \mathrm{gm} /$ $\mathrm{L}=0 ;<=35 \mathrm{gm} / \mathrm{L}=1)$ and serum CRP $(<5 \mathrm{mg} / \mathrm{L}=0 ;>5 \mathrm{mg} /$ $\mathrm{L}=1$ ), GPS score was assigned (minimum 0 ; maximum 2). Survival duration, to a maximum follow-up of 3 months, was measured. Survival proportions were compared for GPS scores of 0,1 and 2 .

Results Total number of patients - 106. Women comprised $52 \%$. Mean age was 73.6 years. Common cancers: lung $(n=26)$, urology $(n=14)$, breast $(n=12)$ and colorectal, upper gastrointestinal and hepatobiliary-pancreas ( 9 each). GPS could be calculated for 79: 0 in one patient, 1 in 33 and 2 in 45 . Whereas $36 \%$ patients with GPS of 1 died during the admission, $47 \%$ of those with GPS of 2 died. At 3 months respective figures were $42 \%$ and $62 \%$. Mean survival was 17.69 days with GPS 2; and 28.20 days with GPS 1 . The sole patient with GPS 0 died during the inpatient stay.

Conclusions Higher GPS is associated with shorter survival in unselected metastatic cancer patients. This study is too small to determine sensitivity and specificity. A larger study for this inexpensive, widely available and easy to use tool is required.

\section{P-82 WHAT LEVEL OF MEDICAL INPUT DO HOSPICE INPATIENTS NEED AND DOES THIS CORRESPOND TO THEIR NEED FOR SPECIALIST NURSING INPUT? A SERVICE EVALUATION IN A UK HOSPICE}

${ }^{1,2}$ Craig Gannon. ${ }^{1}$ Princess Alice Hospice, Esher, UK; ${ }^{2}$ University of Surrey, Guildford, UK

\subsection{6/bmjspcare-2017-00133.81}

Introduction Though medical input to hospice inpatients is well-established, the evidence detailing the nature and level of medical staffing is lacking. Moreover, there are calls for hospices to develop less medical, more public health models.

To facilitate nursing skill-mix changes, and broaden our 'reach' (to include 'low-complexity' patients), our inpatient unit was split equally into generalist nursing (GN) and specialist nursing ( $\mathrm{SN}$ ) beds. It was suggested $\mathrm{GN}$-suitable patients would correspondingly have few medical needs. To facilitate medical workforce planning, we wanted to evaluate the medical needs across these potentially contrasting populations.

Methods We developed a tool to detail the nature and intensity of medical interventions. We completed a 1 month prospective pilot, in a 28-bedded UK hospice, scoring perceived patient need each day.

Results A tool reflecting overall medical need was generated; with 3-ratings (low, moderate, high), across 9 items (e.g. urgency, clinical complexity, trajectory, discord).

284 patient assessments were completed (100\%); the range of medical need for patients in GN beds was; low $=78$, moderate $=41$, high $=14$ and for $\mathrm{SN}$ beds; low $=41$, moderate $=63$, high $=46$. Concordance of medical and nursing complexity for GN patients $=58 \%$ and SN patients $=31 \%$

Discussion A spectrum of need for medical input to hospice inpatients was confirmed; the level fluctuated during a patient's stay and high needs were not restricted to $\mathrm{SN}$ patients.

The limited concordance between a patient's perceived need for medical input and their suitability for SN or GN, questioned the wider applicability of this differentiation. There was a trend for lower medical input for ' $\mathrm{GN}$ ' compared to 'SN patients'. However, 41\% of 'GN patients' had moderate or high medical needs and only $31 \%$ of 'SN patients' had high medical needs.

The pilot tool appeared suitable for benchmarking the need for medical input; informing our workforce planning and warranting further evaluation, to include other care settings. 